\title{
Tissue factor activity on microvesicles from cancer patients
}

\author{
Fanny Ender ${ }^{1}$ - Annika Freund ${ }^{1} \cdot$ Tabea Quecke $^{1}$. Corinna Steidel ${ }^{1} \cdot$ Piet Zamzow $^{1}$ - Nikolas von Bubnoff ${ }^{1}$. \\ Frank Gieseler ${ }^{1} \mathbb{D}$
}

Received: 16 September 2019 / Accepted: 31 October 2019 / Published online: 16 November 2019

(C) The Author(s) 2019

\begin{abstract}
Purpose The expression of active tissue factor (TF) on the surface of microvesicles (MVs) is essential for the activation of the coagulation system and transduction of the signaling pathways in cancer cells. In its use as a biomarker for cancer-associated venous thromboembolism (VTE), TF has shown high expression variability. As a contribution to this discussion, we present a study investigating plasma samples from patients with various progressive tumors at high risk for VTE.

Methods Based on our previous study uncovering microvesicles (MVs), the larger ectosome-like extracellular vesicles (EV), as the major source of TF activity in EV preparations, we now determined TF activity on enriched MVs isolated from plasma of cancer patients and compared it with that on MVs from healthy individuals.

Results We found considerably higher amounts of MVs as well as higher levels of MV-bound TF activities in the plasma of cancer patients. We also show that preparations from plasma of cancer patients have the potency to induce ERK phosphorylation in a human tumor cell line through proteinase-activated receptor two (PAR2) activation.

Conclusion We suggest that MVs instead of whole EV preparations, and TF activity rather than its antigenic quantification should be used in clinical studies for identifying patients with progressive tumors at high risk for VTE.
\end{abstract}

Electronic supplementary material The online version of this article (https://doi.org/10.1007/s00432-019-03073-0) contains supplementary material, which is available to authorized users.

Frank Gieseler

frank.gieseler@uksh.de

1 Clinic for Hematology and Oncology, Section Experimental Oncology, University Hospital od Schleswig-Holstein,

UKSH, Campus Luebeck, Ratzeburger Allee 160,

23528 Luebeck, Germany 


\section{Graphic abstract}

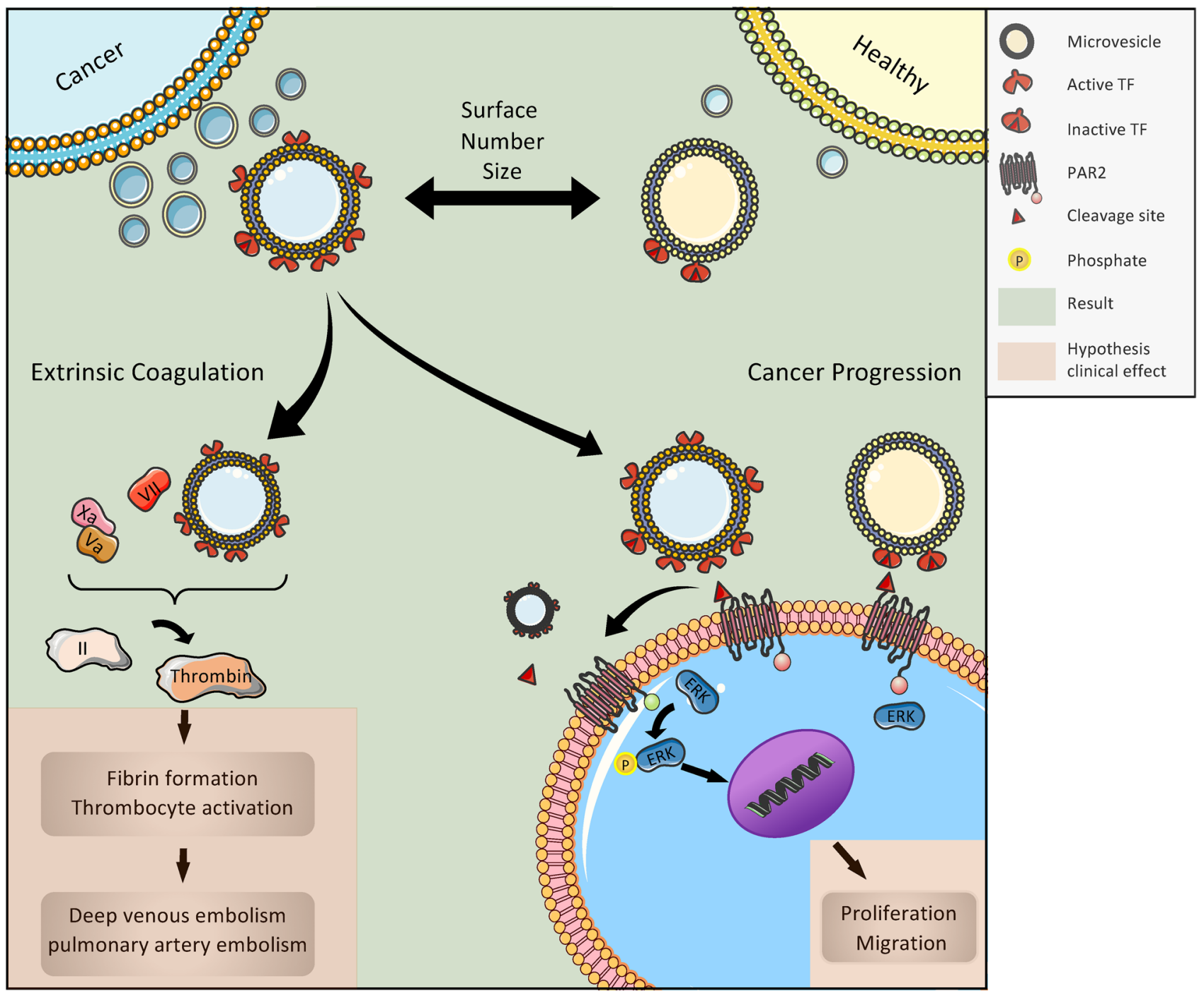

Keywords Extracellular vesicles $\cdot$ Tissue-factor pathway $\cdot$ Progressive cancer

\section{Abbreviations}

TF Tissue factor

MVs Microvesicles

VTE Venous thromboembolism

EVs Extracellular vesicles

PAR2 Proteinase-activated receptor 2

SEM Scanning electron microscopy

TFPI Tissue factor pathway inhibitor

\section{Introduction}

The development of new flow cytometers with resolution capabilities for sub-micron particles has enabled visualization and characterization of small vesicle subpopulations. In principle, two different forms of extracellular vesicles (EVs) can be distinguished: smaller exosomes $(30-100 \mathrm{~nm})$ formed intracellularly within multi-vesicular bodies and released after fusion with the plasma membrane, and larger microvesicles (MVs) $(50-1000 \mathrm{~nm})$ directly arising by outward budding and fission of the plasma membrane (Van Niel et al. 2018). To date, consensus has not yet emerged on specific markers of EV subtypes. Therefore, the International Society for 
Extracellular Vesicles has suggested an EV nomenclature that refers to physical characteristics, biochemical composition, or descriptions of conditions or cellular origin (Théry et al. 2018). In this study, we separated EV subsets through sequential centrifugation based on size and refer to larger vesicles as MVs.

Formerly, it was suggested that EVs were a kind of cellular garbage, but now we know that EVs are formed and released by almost every cell in a directed and highly regulated process (Colombo et al. 2014; Yanez-Mo et al. 2015). They can convey intercellular signals; tumor cells, in particular, and also cells of the immune system use EVs to modulate the surrounding microenvironment through targeted signals, such as activation of the proteinaseactivated receptor-2 (PAR2)/ERK pathway (Robbins and Morelli 2014).

Tumor cells release EVs that present tissue factor (TF), which is able to systemically activate the extrinsic tenase of the coagulation system through the TF pathway (Tesselaar et al. 2007). As this phenomenon can be observed in vitro by using isolated EVs from tumor cell lines and adding plasmatic coagulation factors, clinical studies aim at correlating tissue factor-positive $\mathrm{EVs}\left(\mathrm{TF}^{+} \mathrm{EVs}\right)$ with clinical events such as venous thromboembolism (VTE) (Geddings and Mackman 2013). The clinical background is the high rate of VTE, a major life-threatening event, in patients with progressive cancers. As this pathway can be effectively inhibited, e.g. by using low molecular weight heparins such as tinzaparin (Gamperl et al. 2016), the use of surrogate parameters that indicate a high risk of VTE for the individual patient is warranted. Several studies have shown that increased levels of $\mathrm{TF}^{+} \mathrm{EV}$ s correlate with VTE in patients with cancer (Geddings and Mackman 2013; Khorana et al. 2008). Nevertheless, these correlations are not uniformly found over all cancer types (Thaler et al. 2012), and the topic is still under debate (Gardiner et al. 2015). As the underlying principle, namely the systemic activation of the coagulation system by $\mathrm{TF}^{+} \mathrm{EVs}$, is basically accepted, one of the reasons for the lack of clinical collation in all cancers might be related to the methodologies used in different studies (Gardiner et al. 2015; Thaler et al. 2012). In this context, it has recently been suggested that functional, rather than quantitative assays, based on FXa generation by purified $\mathrm{TF}^{+} \mathrm{EV}$ s may improve sensitivity for predicting thrombotic risk (Graf and Ruf 2018). In the study presented here, we performed functional TF assays using MVs (Gamperl et al. 2016) isolated from the plasma of patients with various progressive cancers and compared the results with those from healthy donors. We have shown before that MVs are the major source of TF activity in EV preparations (Gamperl et al. 2016). The rationale to choose this patient population is their high VTE risk (Donnellan and Khorana, 2017).

\section{Materials and methods}

\section{Characteristics of healthy donors and cancer patients}

All experiments done on human material are in agreement with the Declaration of Helsinki. All volunteers gave informed consent and the study was approved by the Ethics Committee of the University of Luebeck (file number 15-320). Plasma was obtained from 28 healthy donors without any disease and 28 patients suffering from various, advanced and metastatic solid tumors with a high risk to develop VTE. The average age of healthy donors was $29 \pm 5$ years ( $60 \%$ females, $40 \%$ males). The reason for choosing a younger group as negative control is given in the discussion.

The cancer patients in our study were $70 \pm 14$ years old ( $40 \%$ females, $60 \%$ males) and characterized by an advanced tumor stage (stage IV), as tumors had metastasized in $93 \%$, and patients had developed resistance to previous anti-cancer treatment. As patients with progressive cancers, all these belong to the high-risk group for developing VTE (Donnellan and Khorana 2017). The group included patients with cancers of the gastrointestinal tract $(50 \%)$, genitourinary tract $(17 \%)$, female breast $(10 \%)$, respiratory tract $(10 \%)$, hematologic-immunologic system (4\%), sarcoma (3\%), neuroendocrine system (3\%), and cancers of unknown primary (CUP) (3\%).

\section{Isolation of EVs from plasma of healthy donors and cancer patients}

Blood from healthy donors and from patients with advanced stage solid tumors was collected into $7.5 \mathrm{~mL}$ lithium-heparin tubes, $2.9 \mathrm{~mL}$ citrate tubes and $2.6 \mathrm{~mL}$ EDTA tubes (Sarstedt, Nuremberg, Germany). Samples were centrifuged at $470 \times g$ for $10 \mathrm{~min}$ to remove cells and cellular debris. This "platelet-poor plasma" was kept stored at $-20{ }^{\circ} \mathrm{C}$ until used. After thawing, EVs were isolated as described before (Gamperl et al. 2016): samples were centrifuged at $2500 \times g$ for $15 \mathrm{~min}$ at $4{ }^{\circ} \mathrm{C}$, decanted and centrifuged again at $2500 \times g$ for another $15 \mathrm{~min}$. This low-speed centrifugation protocol is in accordance with the recommendation of the International Society on Hemostasis and Thrombosis (ISTH) and removes cellular debris and larger EVs such as apoptotic bodies and oncosomes (Stagnara et al. 2012). For separation of MVs from smaller exosome-like EVs as well as soluble factors such as interleukins and growth factors, a high-speed centrifugation step at $10,000 \times g$ for $90 \mathrm{~min}$ at $4{ }^{\circ} \mathrm{C}$ was performed as described before (Lacroix et al. 2013; Muralidharan-Chari 
et al. 2009). MV-containing pellets were resuspended in PBS for further analysis.

\section{MV quantification by high-resolution flow cytometry}

Samples were analyzed using the high-resolution flow cytometer Cytoflex $S$ (Beckman Coulter, Krefeld, Germany) that enables detection of particles as small as $150 \mathrm{~nm}$ in diameter (Spittler 2015). Set-up and configuration were performed as recommended by the manufacturer for the measurement of EVs. Thereby, the violet side scatter (VSSC) 405/10 served as trigger channel. Analysis was performed until 100,000 events were collected for each tube or at least for $3 \mathrm{~min}$. The flow rate was set to $10 \mu \mathrm{L} /$ $\min$.

\section{Scanning electron microscopy}

Isolated MVs were fixed with glutaraldehyde at a final concentration of $2.5 \%$ in filtered PBS and stored at $4{ }^{\circ} \mathrm{C}$ until further preparation. After homogenization, $10-20 \mu \mathrm{L}$ of each sample was placed onto a Thermanox coverslip (Thermo Fisher Scientific, Darmstadt, Germany) and allowed to settle for 90-120 min in a humid chamber to prevent drying. For dehydration, the samples were then placed into solutions with increasing acetone concentration (70-100\%) and subsequently fully dried via critical point drying using $\mathrm{CO}_{2}$ to avoid shrinkage effects and loss of structure from air-drying. Dehydrated samples were sputtered with gold and analyzed with an EVO LS 15 scanning electron microscope (Zeiss, Germany).

\section{Determination of TF activity on the surface of MVs}

Tenase activity of TF-bearing MVs was determined using the Zymuphen MP-TF Assay (Aniara, West Chester, OH, USA). The wells of the 96-well microplate were coated with a murine, monoclonal anti-human TF antibody, which does not interfere with TF activity. TF-positive MVs within the samples bound to the antibody during an overnight incubation. TF concentration was determined by the potency to activate factor $\mathrm{X}$ after the addition of factor VIIa and factor $\mathrm{X}$. The resulting TF/factor VIIa complex cleaved factor $\mathrm{X}$ into factor Xa (FXa). In a third step, a specific chromogenic FXa substrate was added, which reacted with FXa leading to a substrate turnover. The absorbance, measured at $405 \mathrm{~nm}$ on a photometer, was directly proportional to the amount of $\mathrm{TF}$ presented on MVs in the samples. Samples were measured in quadruplicates.

\section{Cell culture}

All cell-based assays were performed using the pancreas carcinoma cell line COLO 357. These cells, originating from a metastasis of a human pancreatic adenocarcinoma, were established and first described by Morgan et al. (Morgan et al. 1980). The cell line grows as an adherent monolayer and has a rather short cell-doubling time of $21 \mathrm{~h}$. It was chosen because of its stable expression of PAR1 and PAR2 and its low spontaneous migratory capacity (Meitner et al. 1983). Cells were cultured under serum-free conditions with $10 \%$ panexin (Pan Biotech, Aidenbach, Germany) as serum substitute. We decided to use panexin rather than fetal calf serum, due to the large amount of EVs that can be found in fetal calf serum that would interfere with the experiments (Théry et al. 2006).

\section{Analysis of MV-induced cell signaling}

The potency of TF-bearing MVs to activate the ERK 1/2 signaling pathway via PAR2 was determined using the cell-based human ERK1/2 (Thr202/Tyr204) phosphorylation ELISA (RayBiotech Inc., Norcross, GA, USA). COLO 357 cells were seeded into the wells of a 96-well microtiter plate $24 \mathrm{~h}$ prior to use. Three hours before stimulation with MVs, cells were set to starving medium containing only $1 \%$ panexin. Thereafter, cells were incubated with isolated plasma MVs for $10 \mathrm{~min}$. After stimulation, the assay was performed according to the manufacturer's instructions: briefly, cells were fixed, quenched and blocked before incubation with a murine monoclonal antihuman ERK1/2 antibody or a murine monoclonal anti-human phosphoERK1/2 antibody. Staining was performed using a secondary anti-mouse $\mathrm{IgG}$ antibody coupled to horseradish peroxidase (HRP). After administration of the HRP's substrate TMB, the substrate turnover, measured at $450 \mathrm{~nm}$, was proportional to the amount of phosphorylated or unphosphorylated ERK.

\section{Statistical analysis}

All experiments were performed at least in triplicate. Results are shown as individual data points and were analyzed using mean and standard deviation. To test the statistical difference between groups, Student's $t$ test for unequal variances was used. We further evaluated correlations between analyzed parameters by computing Pearson's correlation coefficient (Microsoft Excel for Mac 16.16.8, GraphPad Prism 8.1.1, San Diego, CA, USA). 


\section{Results}

\section{High number of MVs in the plasma of patients with metastasized tumors}

Plasma MVs from healthy donors and cancer patients were detected and quantified by high-resolution flow cytometry. As a size reference, we used Megamix beads (Biocytex, Marseille, France), which comprise populations with different diameters including $0.5,0.9$ and $3 \mu \mathrm{m}$ (Fig. 1a). For the identification of MVs in the size range of $0.5-1 \mu \mathrm{m}$, we applied a respective size gate based on the Megamix beads and counted all events within that gate as MVs (Fig. 1b). Using flow cytometry (Fig. 1b) and scanning electron microscopy (Fig. 1d), we could confirm a selective enrichment of larger vesicles heterogeneous in size by our sequential centrifugation protocol. The total count of plasma MVs from healthy donors and cancer patients is shown in Fig. 1c. In healthy donors, we found an MV concentration of $0.68 \times 10^{5} \pm 1.03 \times 10^{5} \mathrm{MV} /$ $\mathrm{mL}$ plasma; in patient plasma, the MV concentration was $1.73 \times 10^{5} \pm 3.33 \times 10^{5}$. We also found a very high inter-individual difference, especially in cancer patients with a range of two log steps, which prevented statistical significance.

\section{TF activity levels of plasma MVs from cancer patients are significantly higher as compared to healthy donors}

The presentation of $\mathrm{TF}$ is a clinically and functionally relevant characteristic of MVs, as it defines them as potent activators of the extrinsic coagulation system and other cellular signaling pathways such as PAR2/ERK. However, if TF antigen is detected on the surface of MVs, it does not necessarily mean that it is functionally active as previously discussed (Graf and Ruf 2018). Therefore, we measured tenase activity
A

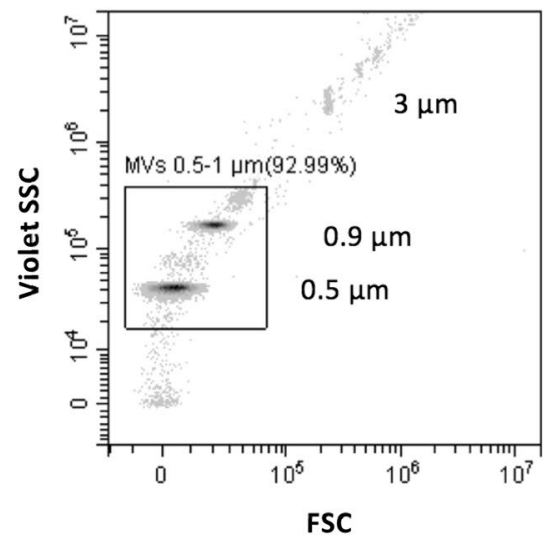

B

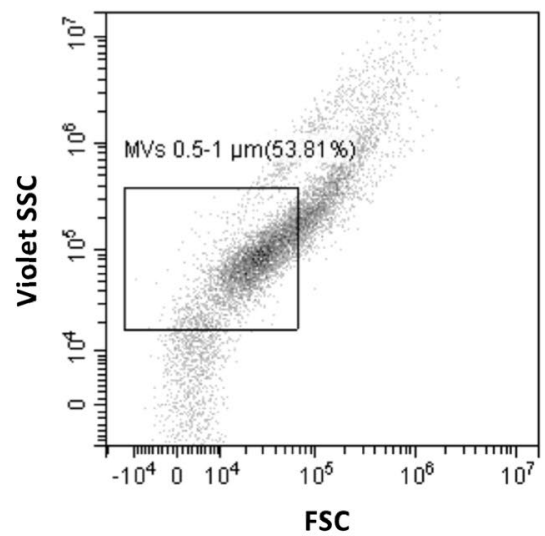

C

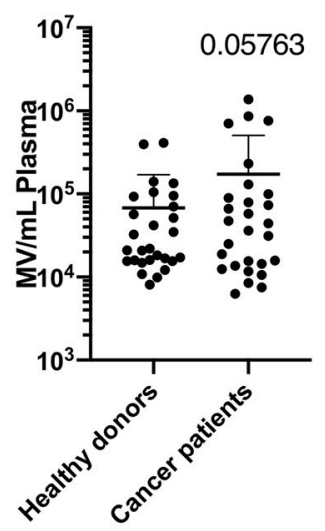

D

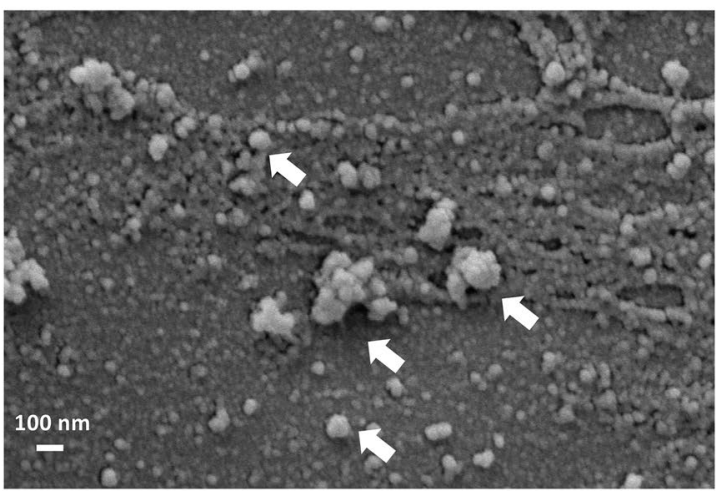

Fig. 1 Microvesicles (MVs) in the plasma of patients with progressive cancers and in healthy donors in high-resolution flow cytometry. a Localization of Megamix beads for size calibration. Bead populations with distinct diameters $(0.5,0.9,3 \mu \mathrm{m})$ are detectable through violet side scatter triggering and presented as density plot. b Plasma MVs identified by high-resolution flow cytometry. Events within the size range of $0.5-1 \mu \mathrm{m}$ were defined as MVs. c Quantification of plasma MVs in the size range of $0.5-1 \mu \mathrm{m}$ from healthy donors and cancer patients. Shown is the mean $\pm \mathrm{SD} ; n=28$. Due to the high inter-individual variance, especially in cancer patients, the differences did not reach statistical significance $(p=0.05763)$. d Characterization of extracellular vesicles by SEM 


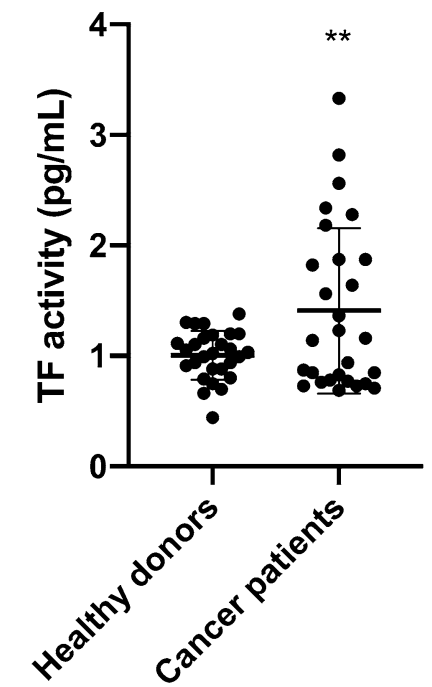

Fig. 2 Tissue factor (TF) activity of plasma MVs from healthy donors and cancer patients. Shown is the mean $\pm \mathrm{SD} ; n=28 ; * * p<0.01$. Note the high inter-individual variance in cancer patients

of TF-bearing MVs using the Zymuphen MP-TF assay as described above. Plasma MVs from healthy donors had a $\mathrm{TF}$ (with tenase activity) concentration of $1.01 \pm 0.22 \mathrm{pg} /$ $\mathrm{mL}$, whereas plasma MVs from patients with advanced tumors showed a significantly higher level of TF activity of $1.41 \pm 0.75 \mathrm{pg} / \mathrm{mL}(p<0.01)$ (Fig. 2).

\section{TF-bearing MVs from cancer patients are potent inducers of the ERK signaling pathway in tumor cells}

The presentation of TF on the surface of MVs is not only important for the activation of extrinsic coagulation but also for that of surface receptors, such as PAR2, on target cells, which leads to the induction of intracellular signaling and subsequently influences cellular functions (Geddings and Mackman 2013). We investigated the potential of plasma MVs to induce ERK phosphorylation in the human pancreatic carcinoma cell line COLO 357. Plasma MVs from cancer patients triggered significantly higher ERK phosphorylation as compared to MVs from healthy donors. Of note, plasma MVs induced ERK phosphorylation in the same range as a specific PAR2 agonist (Fig. 3; controls are shown in supplemental Fig. 1).

\section{Direct correlation between TF activity and ERK phosphorylation of MVs from healthy donors and cancer patients}

To determine functional properties on MV levels, we normalized TF activity and ERK phosphorylation to $10^{6} \mathrm{MVs}$ and found a linear correlation between pERK and TF activity with a correlation coefficient $>0.8$ for plasma MVs of

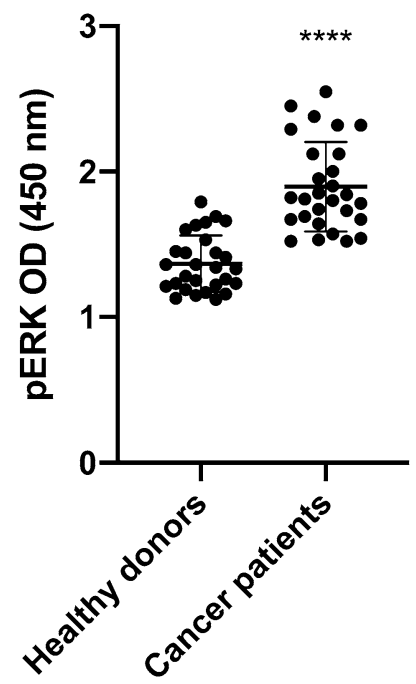

Fig. 3 Plasma-MV mediated induction of ERK phosphorylation in COLO 357 human pancreas carcinoma cells after 10 min of incubation. Shown is the mean $\pm \mathrm{SD} ; n=28 ; * * * * p<0.0001$

both healthy donors and cancer patients (Fig. 4). Of note, the magnitude of a basal ERK phosphorylation induced by plasma MVs is comparable to that induced by a specific PAR2 agonist (supplemental Fig. 1) suggesting that cellular activation occurs via an the PAR2/ERK signaling axis as previously suggested (Gieseler et al. 2013; Graf and Ruf 2018; Ruf et al. 2011).

\section{Discussion}

$\mathrm{TF}^{+} \mathrm{EVs}$ are a major source of blood-borne TF in the context of inflammation and malignancy, thus being potentially responsible for the activation of the coagulation system and subsequently high VTE rate in cancer patients (Date, Eitan et al. 2017). Although clinical studies have shown that increased levels of $\mathrm{TF}^{+} \mathrm{EV}$ s in principle correlate with venous thrombosis in patients with cancer (Geddings and Mackman 2013; Khorana et al. 2008), these correlations are not uniformly found over all cancer types (Thaler et al. 2012). In fact, huge variances of $\mathrm{TF}^{+} \mathrm{EV}$ numbers as well as variations of VTE rates are found in clinical studies, indicating pathophysiological differences between different forms of cancer and patients with comorbidities as well as medication affecting EVs. Cellular membrane expression of TF has been shown on the majority of tumors and it has been linked to the promotion of hematogenous metastasis (Mueller et al. 1992) and the balance of angiogenic and antiangiogenic properties of tumor cells (Zhang et al. 1994). As MVs belong to the EV sub-group of ectosomes, they are formed by outward budding and fission of the plasma membrane, thus representing the membrane composition of their parent 

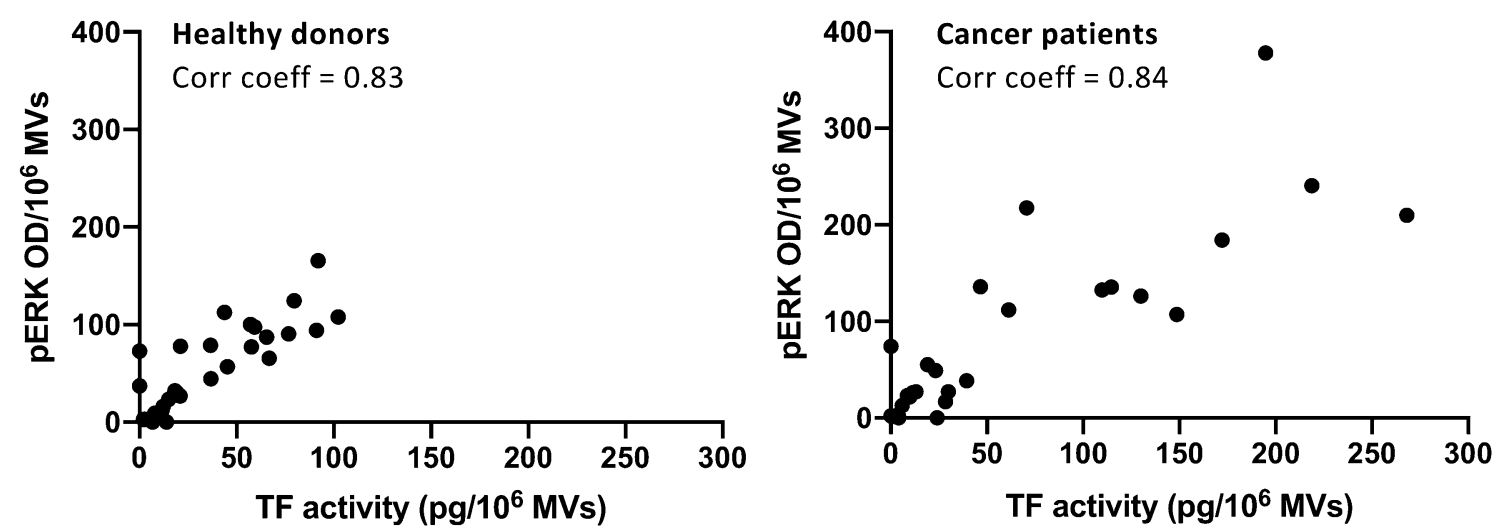

Fig. 4 Direct correlations between TF activity and ERK phosphorylation of MVs isolated from healthy donors (left) and cancer patients (right). TF activity and ERK phosphorylation was normalized to $10^{6}$ MVs. Pearson's correlation coefficients $>0.8$

cells (Van Niel et al. 2018). Therefore, in principle, they could be used as liquid biopsies in the diagnosis of cancer (Ender et al. 2019; Mathai et al. 2019).

A number of different co-factors such as protein disulfide isomerase (PDI) or the co-exposure of phosphatidylserine on the outer layer of cell membrane modulate TF activity (Popescu et al. 2010). In addition, it has been proposed that the expression of TF isomers, namely encrypted or decrypted TF, with only the latter significantly contributing to extrinsic tenase activity (TF-FVIIa), might be responsible for the variations of TF activity (Graf and Ruf 2018). Therefore, in this study we performed assays that determined TF activity rather than TF concentration on MVs (larger ectosome-like vesicles) isolated from patients with progressive cancers. Using sequential centrifugation steps including high-speed centrifugation, we enriched MVs to achieve more uniform results. In order to judge the results, we have compared them to those from healthy donors without evidence of thrombosis. For this study, we decided to choose a true negative population of younger age, with no signs of any disease including thrombosis, and no medication, since age-matched controls would presumably suffer from co-morbidities, risk factors for age-related diseases and poly-medication. The best control group would have been the same patient before and after cancer diagnosis, which is not possible. The issue with people at higher age is heterogeneity regarding co-morbidities potentially influencing the number and structure of EVs (Extermann 2000).

Plasma EVs show age-related changes that affect not only the number but also the structure and content of the vesicles. Although senescent cells release more EVs (Robbins and Morelli 2014), plasma EV concentration decreases with advancing age, probably because EVs from older individuals are more readily internalized by $\mathrm{B}$ cells (Eitan et al. 2017). EVs activate both monocytes and B cells, and activation of B cells by lipopolysaccharide (LPS) enhances EV internalization (Eitan et al. 2017). As our results showed more EVs in older patients (Fig. 1c), we assume that this effect is disease related and not just a matter of aging. Using flow cytometry or SEM for the characterization of plasma MVs, we could show that sequential centrifugation leads to selective enrichment of larger vesicles heterogeneous in size. Notably, determining the actual size of plasma EV preparations after high-speed centrifugation led to different results depending on the method that was used. While we identified particle sizes up to $3 \mu \mathrm{m}$ by flow cytometry and the use of Megamix beads with defined size populations (Fig. 1b), we could identify particles within a size range of $50-500 \mathrm{~nm}$ by SEM (Fig. 1d). This discrepancy can be linked to the properties of the polystyrene beads we used for size calibration. We are aware that the refractive index of these beads differs from those of cells and EVs (Chandler et al. 2011; Mullier et al. 2011; Robbins and Morelli 2014). Lipid-based vesicles have a lower refractive index and hence, scatter much less light than polystyrene beads, which might influence size determination. Importantly, we found that Megamix bead-based size determination of $\mathrm{EV}$ preparations by flow cytometry seems to overestimate particle sizes.

Although the increased MV levels in the plasma of patients with progressive cancers did not reach the level of statistical significance when compared to healthy donors, the inter-individual variance of MV levels was much higher in cancer patients than in healthy donors overlapping two log-ranges (Fig. 1c). This was also the case for TF activity, which was significantly higher in MVs from the plasma of cancer patients as compared to healthy donors (Fig. 2). These results reflect our analysis of a real-life group of patients treated in our hospital. Given our choice of a control group of healthy young donors, the observed variances are not surprising. These observations are per se remarkable but obviously not sufficient to explain the difference in VTE rates in different cancers. One of the factors that increase the 
release of EVs is tumor cell stimulation with inflammatory cytokines and endotoxin (Yamamoto et al. 2015). Hence, determining the concentration of EVs might be a promising tool in clinical studies to determine the VTE risk of patients. To show that $\mathrm{TF}^{+} \mathrm{MVs}$ are biologically active, we performed ERK phosphorylation assays using human pancreatic carcinoma cells COLO 357. Although the MAPK/ ERK pathway can be activated by several stimuli, it is one of the elemental signaling systems that controls fundamental cellular processes as proliferation, differentiation, survival and apoptosis (Sun et al. 2015). In Fig. 3, it is shown that MVs isolated from cancer patients have a significant higher potency to induce cellular ERK phosphorylation as plasma MVs from healthy donors. On the MV level, we found a direct correlation of TF activity and ERK phosphorylation (Fig. 4), which points to the decisive role of TF activity for the cellular effects of plasma MVs in cancer patients. Using sequential centrifugation for EV purification, we got rid of soluble plasma factors such as cytokines and endotoxin. Thus, plasma MVs resuspended from pellets after high-speed centrifugation are responsible for the described cellular effects.

\section{Conclusions}

The experiments were performed to determine if $\mathrm{TF}^{+} \mathrm{EVs}$ can be used as a surrogate parameter for patients at high risk for VTE. We have shown before that larger MVs are the major source of TF activity in EV preparations (Gamperl et al. 2016). Here we show that patients with progressive tumors being at high risk for VTE have indeed more MVs with significantly higher TF activity. With regard to the high inter-individual variance in the group of older cancer patients, we do not think that $\mathrm{TF}^{+} \mathrm{EV}$ s can be used as a marker for all different types of cancers. Nevertheless, $\mathrm{TF}^{+} \mathrm{EV}$ s can be a surrogate parameter for the risk of developing cancer-associated VTE for individual patients and might indicate the use of low molecular weight heparins with the potency to release TFPI, such as tinzaparin. This effect might be especially beneficial as $\mathrm{TF}^{+} \mathrm{EV}$ s not only activate the coagulation system but also have migrationinducing effects on PAR2-expressing cancer cells (Ender et al. 2019; Gamperl et al. 2016; Gieseler et al. 2013).

Acknowledgements We thank Rajam Csordas for her excellent English editing. We thank Servier Medical Art (smart.servier.com) for providing tools for the graphical abstract. We thank Matthias Klinger and Hristo Örun for performing scanning electron microscopy.

Author contributions Conceptualization, FG; Methodology, FG; Software, FG; Validation, FG, FE and AF; Formal Analysis, FE and AF; Investigation, FE, AF, CS; Resources, FG; Data Curation, FG and FE; Writing, Original Draft Preparation, FG and FE; Writing, Review \&
Editing, all authors; Visualization, FG, FE and PZ; Supervision, FE; Project Administration, FG and FE; Funding Acquisition, FG.

Funding Annelise-Asmussen-Stiftung, Luebeck [grant 180802], LEO Pharma Germany [grant 180208].

\section{Compliance with ethical standards}

Conflict of interest The authors declare no conflict of interest.

Open Access This article is distributed under the terms of the Creative Commons Attribution 4.0 International License (http://creativeco mmons.org/licenses/by/4.0/), which permits unrestricted use, distribution, and reproduction in any medium, provided you give appropriate credit to the original author(s) and the source, provide a link to the Creative Commons license, and indicate if changes were made.

\section{References}

Chandler W, Yeung W, Tait J (2011) A new microparticle size calibration standard for use in measuring smaller microparticles using a new flow cytometer. J Thromb Haemost 9(6):1216-1224. https:// doi.org/10.1111/j.1538-7836.2011.04283.x

Colombo M, Raposo G, Thery C (2014) Biogenesis, secretion, and intercellular interactions of exosomes and other extracellular vesicles. Annu Rev Cell Dev Biol 30:255-289. https://doi. org/10.1146/annurev-cellbio-101512-122326

Date K, Ettelaie C, Maraveyas A (2017) Tissue factor-bearing microparticles and inflammation: a potential mechanism for the development of venous thromboembolism in cancer. J Thromb Haemost 15(12):2289-2299. https://doi.org/10.1111/jth.13871

Donnellan E, Khorana AA (2017) Cancer and venous thromboembolic disease: a review. Oncologist 22(2):199-207. https://doi. org/10.1634/theoncologist.2016-0214

Eitan E, Green J, Bodogai M, Mode NA, Bæk R, Jørgensen MM, Biragyn A (2017) Age-related changes in plasma extracellular vesicle characteristics and internalization by leukocytes. Sci Rep 7(1):1342. https://doi.org/10.1038/s41598-017-01386-z

Ender F, von Bubnoff N, Gieseler F (2019) Extracellular vesicles: subcellular organelles with the potential to spread cancer resistance. Anticancer Res 39(7):3395-3404. https://doi.org/10.21873/antic anres. 13483

Extermann M (2000) Measurement and impact of comorbidity in older cancer patients. Crit Rev Oncol/Hematol 35(3):181-200. https:// doi.org/10.1016/S1040-8428(00)00090-1

Gamperl H, Plattfaut C, Freund A, Quecke T, Theophil F, Gieseler F (2016) Extracellular vesicles from malignant effusions induce tumor cell migration: inhibitory effect of LMWH tinzaparin. Cell Biol Int 40(10):1050-1061. https://doi.org/10.1002/cbin.10645

Gardiner C, Harrison P, Belting M, Böing A, Campello E, Carter BS, van Es N (2015) Extracellular vesicles, tissue factor, cancer and thrombosis-discussion themes of the ISEV 2014 educational day. J Extracell Vesicles 4(1):26901. https://doi.org/10.3402/jev. v4.26901

Geddings JE, Mackman N (2013) Tumor-derived tissue factor-positive microparticles and venous thrombosis in cancer patients. Blood 122(11):1873-1880. https://doi.org/10.1182/blood-201304-460139

Gieseler F, Ungefroren H, Settmacher U, Hollenberg MD, Kaufmann R (2013) Proteinase-activated receptors (PARs)-focus on receptor-receptor-interactions and their physiological and 
pathophysiological impact. Cell Commun Signal 11(1):86. https ://doi.org/10.1186/1478-811X-11-86

Graf C, Ruf W (2018) Tissue factor as a mediator of coagulation and signaling in cancer and chronic inflammation. Thromb Res 164:S143-S147. https://doi.org/10.1016/j.thromres.2018.01.023

Khorana A, Francis C, Menzies K, Wang JG, Hyrien O, Hathcock J, Taubman M (2008) Plasma tissue factor may be predictive of venous thromboembolism in pancreatic cancer. $\mathrm{J}$ Thromb Haemost 6(11):1983-1985. https://doi.org/10.111 1/j.1538-7836.2008.03156.x

Lacroix R, Judicone C, Mooberry M, Boucekine M, Key NS, DignatGeorge F, Workshop IS (2013) Standardization of pre-analytical variables in plasma microparticle determination: results of the International society on thrombosis and haemostasis SSC collaborative workshop. J Thromb Haemost 11(6):1190-1193. https ://doi.org/10.1111/jth.12207

Lo Cicero A, Stahl PD, Raposo G (2015) Extracellular vesicles shuffling intercellular messages: for good or for bad. Curr Opin Cell Biol 35:69-77. https://doi.org/10.1016/j.ceb.2015.04.013

Mathai RA, Vidya RVS, Reddy BS, Thomas L, Udupa K, Kolesar J, Rao M (2019) Potential utility of liquid biopsy as a diagnostic and prognostic tool for the assessment of solid tumors: implications in the precision oncology. J Clin Med 8(3):373. https://doi. org/10.3390/jcm8030373

Meitner PA, Kajiji SM, LaPosta-Frazier N, Bogaars HA, Jolly GA, Dexter DL, Tumer MD (1983) "COLO 357," a human pancreatic adenosquamous carcinoma: growth in artificial capillary culture and in nude mice. Cancer Res, 43(12 Part 1):5978-5985. PubMed 6640542

Morgan R, Woods L, Moore G, Quinn L, McGavran L, Gordon S (1980) Human cell line (COLO 357) of metastatic pancreatic adenocarcinoma. Int J Cancer 25(5):591-598. https://doi.org/10.1002/ ijc. 2910250507

Mueller BM, Reisfeld RA, Edgington TS, Ruf W (1992) Expression of tissue factor by melanoma cells promotes efficient hematogenous metastasis. Proc Natl Acad Sci 89(24):11832-11836. https://doi. org/10.1073/pnas.89.24.11832

Mullier F, Bailly N, Chatelain C, Dogne J, Chatelain B (2011) More on: calibration for the measurement of microparticles: needs, interests, and limitations of calibrated polystyrene beads for flow cytometry-based quantification of biological microparticles. J Thromb Haemost 9(8):1679-1681. https://doi.org/10.111 1/j.1538-7836.2011.04386.x

Muralidharan-Chari V, Clancy J, Plou C, Romao M, Chavrier P, Raposo G, D'Souza-Schorey C (2009) ARF6-regulated shedding of tumor cell-derived plasma membrane microvesicles. Curr Biol 19(22):1875-1885. https://doi.org/10.1016/j.cub.2009.09.059

Popescu NI, Lupu C, Lupu F (2010) Role of PDI in regulating tissue factor: fVIIa activity. Thromb Res 125:S38-S41. https://doi. org/10.1016/j.thromres.2010.01.034

Robbins PD, Morelli AE (2014) Regulation of immune responses by extracellular vesicles. Nat Rev Immunol 14(3):195. https://doi. org/10.1038/nri3622

Ruf W, Disse J, Carneito-Lobo TC, Yokota N, Schaffner F (2011) Tissue factor and cell signalling in cancer progression and thrombosis. J Thromb Haemost 9:306-315. https://doi.org/10.111 1/j.1538-7836.2011.04318.x
Spittler A (2015) Set-up pf CytoFLEX for extracellular vesicle measurement. Beckman Coulter Application Notes. https://media .beckman.com/-/media/pdf-assets/application-notes/flow-cytom etry-application-note-cytoflex-set-up-extracellular-vesicles.pdf

Spronk HM, ten Cate H, van der Meijden PE (2014) Differential roles of tissue factor and phosphatidylserine in activation of coagulation. Thromb Res 133:S54-S56. https://doi.org/10.1016/j.throm res.2014.03.022

Stagnara J, Garnache Ottou F, Angelot F, Mourey G, Seilles E, Biichle S, Racadot E (2012) Correlation between platelet-derived microparticle enumeration by flow cytometry and phospholipid-dependent procoagulant activity in microparticles: the centrifugation step matters! Thromb Haemost 107(6):1185-1187. https://doi. org/10.1160/TH11-07-0509

Sun Y, Liu W-Z, Liu T, Feng X, Yang N, Zhou H-F (2015) Signaling pathway of MAPK/ERK in cell proliferation, differentiation, migration, senescence and apoptosis. J Recept Signal Transduct 35(6):600-604. https://doi.org/10.3109/10799893.2015.1030412

Tesselaar M, Romijn F, Van Der Linden I, Prins F, Bertina R, Osanto S (2007) Microparticle-associated tissue factor activity: a link between cancer and thrombosis? J Thromb Haemost 5(3):520 527. https://doi.org/10.1111/j.1538-7836.2007.02369.x

Thaler J, Ay C, Pabinger I (2012) Clinical significance of circulating microparticles for venous thrombo-embolism in cancer patients. Hämostaseologie 32(02):127-131. https://doi.org/10.5482/ ha-1164

Théry C, Amigorena S, Raposo G, Clayton A (2006) Isolation and characterization of exosomes from cell culture supernatants and biological fluids. Curr Protocols Cell Biol 30(1):3-22. https://doi. org/10.1002/0471143030.cb0322s30

Théry C, Witwer KW, Aikawa E, Alcaraz MJ, Anderson JD, Andriantsitohaina R, Atkin-Smith GK (2018) Minimal information for studies of extracellular vesicles 2018 (MISEV2018): a position statement of the international society for extracellular vesicles and update of the MISEV2014 guidelines. J Extracell Vesicles 7(1):1535750. https://doi.org/10.1080/20013078.2018.1535750

Van Niel G, D'Angelo G, Raposo G (2018) Shedding light on the cell biology of extracellular vesicles. Nat Rev Mol Cell Biol 19(4):213. https://doi.org/10.1038/nrm.2017.125

Yamamoto S, Niida S, Azuma E, Yanagibashi T, Muramatsu M, Huang TT, Nagai Y (2015) Inflammation-induced endothelial cell-derived extracellular vesicles modulate the cellular status of pericytes. Sci Rep 5:8505. https://doi.org/10.1038/srep08505

Yanez-Mo M, Siljander PR, Andreu Z, Zavec AB, Borras FE, Buzas EI, De Wever O (2015) Biological properties of extracellular vesicles and their physiological functions. J Extracell Vesicles 4:27066. https://doi.org/10.3402/jev.v4.27066

Zhang Y, Deng Y, Luther T, Müller M, Ziegler R, Waldherr R, Nawroth PP (1994) Tissue factor controls the balance of angiogenic and antiangiogenic properties of tumor cells in mice. J Clin Investig 94(3):1320-1327. https://doi.org/10.1172/JCI117451

Publisher's Note Springer Nature remains neutral with regard to jurisdictional claims in published maps and institutional affiliations. 